\title{
Investigating Mechanisms of Radiation-Induced DNA Damage Using Low-Energy Photons
}

\author{
M. Folkard AND K.M. Prise
}

Gray Cancer Institute, Mount Vernon Hospital, P.O. Box 100

Northwood, HA6 2JR, UK

Central to any mechanistic biophysical model of radiation damage to DNA is the relationship between the amount and distribution of energy deposited in the DNA helix and the subsequent production of DNA damage. It is now clear from a number of studies that the minimum energy required to produce bond breaks in DNA is significantly lower than might be expected. For example, some biophysical models have assumed that it takes several 10s of $\mathrm{eV}$ to produce a double-strand break in DNA. However, using low-energy photons, we have shown that energy depositions as low as $7 \mathrm{eV}$ can induce double-strand break and that this is enhanced when the DNA is hydrated, showing that free radical damage is also important.

PACS numbers: 87.50.--a

\section{Introduction}

The study of radiation damage to biomolecules underpins our understanding of the mechanisms of radiation damage to cells, tissues, and living organisms. Theoretical track structure modelling shows us that penetrating radiations (i.e. energetic photons or electrons) produce a significant number of nanometre-sized clusters of ionisations at the low-energy track-ends of secondary electrons (with energy clusters of hundreds of eV, or less) $[1,2]$. Such clusters can induce "complex" strand-breaks in DNA, which are less easily repaired than the predominantly "simple" breaks produced by energetic photons or electrons. The low-energy electrons therefore have an important role in determining overall radiobiological effect of ionizing radiations and the mechanisms by which they damage DNA. Recent studies have indicated that low-energy electrons can induce single-strand breaks (SSB, where one strand of the DNA double-helix is broken) and double-strand breaks (DSB, where both strands are broken) in DNA below the threshold for ionisation, via the formation and decay of molecular resonances involving DNA components 
$[3,4]$. Our own studies [5-7] have endeavoured to quantify the amount of energy involved in the induction of strand-breaks in DNA. The approach we have used is to expose plasmid DNA to low-energy ionizing radiation at a range of energies and look for thresholds, below which SSB and DSB are not produced. The DSB is thought to be the critical damage that leads to lasting damage in cells and ultimately, the host organism. Initial studies were performed using dry DNA in vacuum; however, we have also developed a novel "wet cell" that permits the exposure of DNA in solution to vacuum-UV energies below $10 \mathrm{eV}$. Even at these low energies, we find that SSB and DSB are readily induced in DNA, and that indirect damage through the formation of radicals in the water is an important mechanism.

\section{Methods}

\subsection{Sources of vacuum- $U V$ radiation}

Our initial studies were undertaken at the $2 \mathrm{GeV}$ electron synchrotrons at the Daresbury Laboratory, UK [5, 6]. For studies between $40 \mathrm{eV}$ and $150 \mathrm{eV}$, we used Station 3.3, fitted with a toroidal grating monochromator. For experiments at lower energies (between $7 \mathrm{eV}$ and $25 \mathrm{eV}$ ) Station 3.1 fitted with a Seya-Namioka 1 metre monochromator was used. While synchrotrons are the source of choice (as they offer the widest range of energies) it is also possible to use a "bench-top" UV lamp for low energy exposures. This approach offers a convenient and cost-effective alternative for some studies, therefore we also use this method [7]. Our bench-top irradiator makes use of an RF excited xenon lamp and a concave grating monochromator (Resonance Ltd, Canada) and provides a useful output in the 7-8.5 eV energy range. The arrangement is shown in Fig. 1, fitted with our wet-cell DNA irradiator (described below). For experiments using both the synchrotron

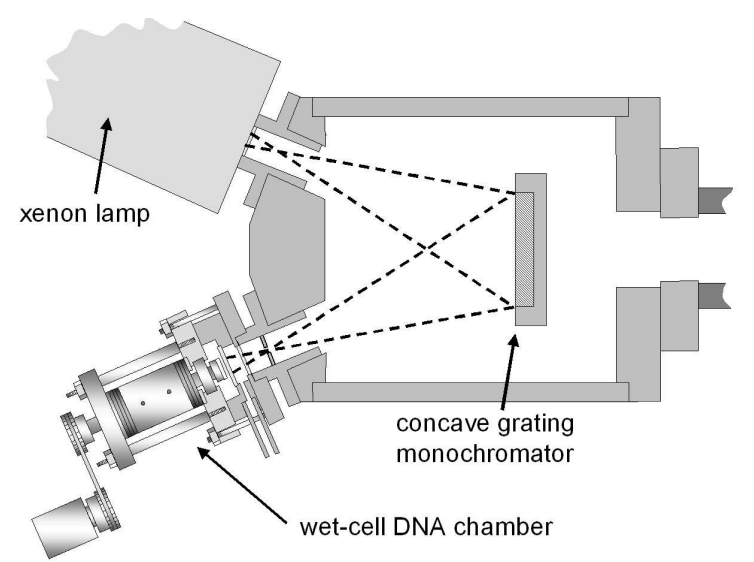

Fig. 1. "Bench-top" exposure system used to irradiate DNA in solution, comprising a xenon UV lamp and a concave grating monochromator fitted with the wet-cell irradiator. 
and the lamp, the photon flux at the sample position was determined using a calibrated silicon photodiode, of area $1 \mathrm{~cm}^{2}$ (type AXUV-100, IRD, Torrance, CA). Using the synchrotron at energies above $40 \mathrm{eV}$, typical exposure rates at the sample position were in the region of $10^{11}-10^{13}$ photons $\mathrm{cm}^{-2} \mathrm{~s}^{-1}$ and useful exposures could be delivered in just a few tens of seconds. At the lowest synchrotron energies however (i.e. $<10 \mathrm{eV}$ ) long exposure times were required, due largely to the lower output of this monochromator. At $7 \mathrm{eV}$, some of the longest exposures were taking up to 8 hours when irradiating dry DNA. Using the xenon lamp, the measured flux at $8.5 \mathrm{eV}$ (where output is greatest) was about $3 \times 10^{10}$ photons $\mathrm{cm}^{-2} \mathrm{~s}^{-1}$ such that exposure times of several tens of minutes were typically required. At $7 \mathrm{eV}$, exposure times were significantly longer, taking 1-2 hours. The reduced exposure times using the xenon lamp arise partly because it was used to irradiate DNA in solution, such that unlike irradiating a dry DNA monolayer, all the energy is transferred to the sample.

\subsection{DNA sample preparation and exposure}

Our target molecule for these studies is extracted and purified plasmid DNA, pBR322 (4363 base-pairs). After exposure to radiation, the plasmid assumes a different topology depending on the damage it receives. Undamaged DNA is in a compact "supercoiled" form, but changes to a "relaxed" form if it received an SSB and a "linear" form following a DSB. The different forms of DNA can be quantified by gel electrophoresis, such that it is relatively straightforward to measure the fraction of induced SSB and DSB [6].

To irradiate dry DNA in vacuum, a DNA monolayer must be prepared. This is achieved by spreading a $1 \mu \mathrm{l}$ sample over a $10 \mathrm{~mm}$ square area of a glass cover slip and freeze-drying the sample. This removes the water without any pile-up of the DNA as the drop evaporates. The sample is then placed in a support at the end of the synchrotron beamline, which uses either a raster-scanning, or rotating arrangement to ensure an even dose distribution over the sample. The dose to the sample is monitored by passing the beam through a fine conducting mesh connected to an electrometer, calibrated previously using the photodiode. To separate the synchrotron vacuum from the sample, selectable UV transmission windows, each mounted in separate window valves, are used. Depending on the UV energy, either a 100-150 nm thick aluminium or polyimide filter is fitted. For studies below $10 \mathrm{eV}$, it is possible to use thick (and therefore, robust) UV transmission windows, such as magnesium fluoride.

In later studies, the arrangement was modified to allow humidified DNA to be irradiated. The DNA was humidified by exposing the evacuated sample chamber to water vapour at a low vapour-pressure (typically $30 \mathrm{mbar}$ ), or if a magnesium fluoride window was used, to humidified helium gas at atmospheric pressure. Full details of the dry and humidified DNA irradiator and sample preparation can be found in Folkard et al. [6]. The use of a thick window has also allowed us to successfully develop a novel "wet cell" which enables the exposure of DNA 
in aqueous solution with UV photons produced using our xenon lamp [7]. The wet cell comprises a chamber with a $1.5 \mathrm{~mm}$ thick magnesium fluoride vacuum window through which the radiation passes. At these energies, the photons have very little penetration in water, therefore a very thin solution must be presented to the radiation beam to maximise the fraction of solution (and hence DNA) that absorbs radiation. A sample is prepared by pipetting $5 \mu$ l of solution containing $250 \mathrm{ng}$ of DNA onto a flat glass disc and trapping it in a $50 \mu \mathrm{m}$ gap between the disc (which rotates to stir the solution) and the non-vacuum side of the magnesium fluoride window (see Fig. 1 and [7]).

\section{Results and discussion}

The induction of SSB and DSB in dry plasmid DNA in the energy range $7-150 \mathrm{eV}$ is shown in Fig. 2. The error bars indicate the standard deviation of repeated experiments; however, there are also significant systematic errors that arise from the difficulty in estimating the breaks per absorbed photon (which critically depends on achieving a good sample monolayer and dose uniformity). Therefore, until more detailed measurements can be made, it is the trends shown in the data that should be noted, rather than the estimated values. From the data, two interesting observations were made. First, it is evident that both SSB and DSB are produced at all energies studied. Second, it can be noted that the shape of the action spectrum is similar for SSB and DSB induction.

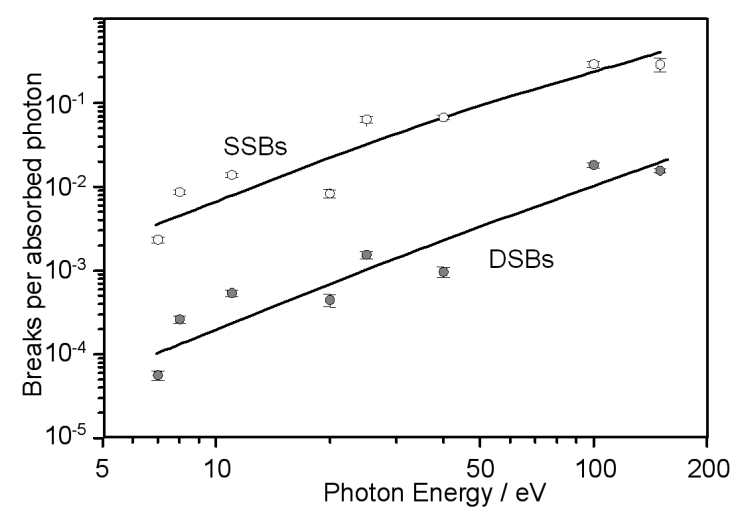

Fig. 2. The induction of SSB and DSB in dry plasmid DNA in the energy range 7-150 eV. The lines are for guidance only.

The observation that DSB were produced reasonably efficiently in dry DNA by photons with energies as low as $7 \mathrm{eV}$ is surprising. This suggests that a single ionization is sufficient to break both strands of the DNA helix, within a few base-pairs of each other. One possibility is that the DSB arise from pairs of SSB separately induced on opposite strands. However, at the exposure levels used in the study, only a few SSB per plasmid are induced, therefore the chance of 
two such breaks occurring close to each other (and on opposite strands) is small. Also, it would be expected that the dose-effect curve for DSB induction would be quadratic in shape, but what is actually observed is a linear relationship. The similarity in shapes for the induction of SSBs and DSBs is also unexpected, and suggests that there is a common precursor for both types of damage, but with a 12-20-fold higher probability of producing a SSB. Both observations suggest that there exists a single-event pathway to DSB induction.

For these experiments, the DNA was in a "dry" state under vacuum. However, in vivo DNA is associated with considerable quantities of bound and free water. It is known that a significant fraction of the damage to DNA by ionizing radiations is "indirect" due to hydroxyl radicals generated in the water. Clearly, it is important to have water present during the irradiation if we are to understand the indirect effect. As explained above, we modified our synchrotron-based irradiator so that either humidified helium gas (for experiments below $10 \mathrm{eV}$ ) or water vapour could be introduced during the exposure to re-hydrate the DNA. When re-hydrated, we see a 2 to 5 -fold increase in sensitivity in both single-strand and double-strand break induction, although some variation between experiments is observed (thought to be due to insufficient control over the extent to which the DNA is re-hydrated). This increase in sensitivity is most likely due to the additional indirect effect of radicals produced in the hydration layer surrounding the DNA, although it is also possible that charge-transfer processes from water tightly-bound to the DNA are also occurring. However, it is known that this water layer is difficult to remove and therefore may also be present in our so-called "dry" DNA samples.

Our most recent data has extended our hydrated studies by using the wet-cell arrangement for irradiating DNA in solution. Figure 3 shows the loss of supercoiled DNA and the formation of linear DNA after irradiation of DNA in solution by $7 \mathrm{eV}$ photons. The dose value is derived from the measured photon flux and the known mass of sample (DNA, plus solution). There is an uncertainty of $10-20 \%$ in the dose value due to observed changes in the response of the diode used for calibration, probably as a result of surface contamination.

In general, our experiments using this arrangement indicate a large increase in the yield of strand breaks when compared to the effects of exposures in the absence of water. This might be expected as significant radical damage can occur in the aqueous system. The addition of a radical scavenger appreciably reduces the amount of SSB and DSB damage induced at all dose levels, showing that $\mathrm{OH}$ radical damage is a significant pathway at these energies [7]. The data shown in Fig. 3 indicate a linear DSB induction as a function of dose. This is indicative of a single-event mechanism, and together with the study using a scavenger, suggests that a single $\mathrm{OH}$ radical has a significant probability of inducing a DSB. Overall, these data point to single-event induction of DSB being a significant pathway with all radiation types. 

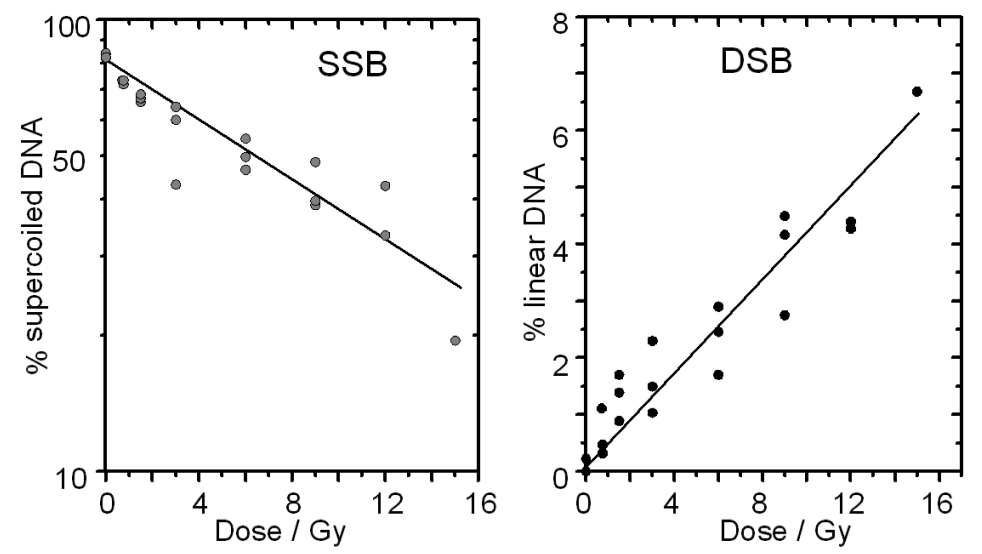

Fig. 3. The loss of supercoiled DNA and the formation of linear DNA after irradiation of DNA in solution by $7 \mathrm{eV}$ photons.

In recent years, it has become increasingly evident that interaction mechanisms other than ionization may have an important role with regard to radiation interaction with tissue. Specifically, recent studies have been reported using a low-energy electron irradiator capable of irradiating plasmid DNA under UHV conditions with electrons down to fractions of an $\mathrm{eV}[3,4]$. In these studies, it was found that SSB and DSB are readily induced by radiations with energies below the ionization limit. Unlike our studies using photons however, they find a significant amount of structure in the energy dependence of induced damage below $15 \mathrm{eV}$. In particular, they observe a peak at about $10 \mathrm{eV}$. Another difference is that their measured ratio for SSB to DSB induction is about 4:1 which is appreciably less than our data for photons. They attribute the strong energy dependence of the DNA breaks to electron attachment within the DNA molecule, followed by localized bond rupture and subsequent reactions of the fragment products. The attachment of an electron leads to the formation of a transient molecular anion state, which can decay by electron auto-detachment, or by dissociation. This can lead to the formation of a single-strand break. The observation of double-strand breaks at these low energies suggests that some fragmentation products can migrate and react with the opposite strand.

\section{Conclusions}

It is now evident that the abundance of low-energy electrons set in motion by all ionizing radiations play a crucial role in DNA damage, but it is still unclear to what extent the various mechanisms by which the electrons break bonds in DNA are important and the energy ranges over which they may apply. An important step forward would be to fully establish meaningful yields of DNA breakage that could eventually serve to build a quantitative mechanistic model that relates the 
initial energy deposition to the observed incidence of single- and double-strand breaks.

\section{References}

[1] H. Nikjoo, P. O’Neill, M. Terrissol, D.T. Goodhead, Int. J. Radiat. Biol. 66, 453 (1994).

[2] H. Nikjoo, P. O'Neill, D.T. Goodhead, M. Terrissol, Int. J. Radiat. Biol. 71, 467 (1997).

[3] B. Boudaiffa, P. Cloutier, D. Hunting, M.A. Huels, L. Sanche, Science 287, 1658 (2000).

[4] F. Martin, P.D. Burrow, Z. Cai, P. Cloutier, D. Hunting, L. Sanche, Phys. Rev. Lett. 93, 068101 (2004).

[5] K.M. Prise, M. Folkard, B.D. Michael, B. Vojnovic, B. Brocklehurst, A. Hopkirk, I.H. Munro, Int. J. Radiat. Biol. 76, 881 (2000).

[6] M. Folkard, K.M. Prise, B. Vojnovic, B. Brocklehurst, B.D. Michael, Int. J. Radiat. Biol. 76, 763 (2000).

[7] M. Folkard, K.M. Prise, C.J. Turner, B.D. Michael, Radiat. Prot. Dosimetry 99, 147 (2002). 